\title{
SPECTRUM OF ACUTE KIDNEY INJURY IN THE MEDICINE WARDS OF A TERTIARY CARE HOSPITAL IN GOA
}

\section{Dr. Ana Soraya Monteiro}

\section{Dr. Edwin Gomes}

\section{Senior resident, Department of Medicine, Goa Medical College, Bambolim} Goa

Professor and Head, Department of Medicine, Goa Medical College, Bambolim Goa

Junior resident, Department of Medicine, Goa Medical College, Bambolim Goa *Corresponding Author

ABSTRACT Background: Acute kidney injury is characterized by an increase in serum creatinine and/or a decrease in urine output. It can be classified as pre-renal, renal, or postrenal in cause and can be attributed to a local or systemic event - due to direct injury to the kidney or through indirect impediment of renal function.

Objective: To determine the prevalence and spectrum of acute kidney injury in the medicine wards of a tertiary health care centre in Goa.

Method: A case series was conducted on 100 patients admitted for AKI, or developed it during the hospital stay.

Results: AKI was more common in males, with a male to female ratio of 3.5:1, and more prevalent in the age groups of $25-34$ and 45-64 years. The dialysis requirement was $28 \%$ and mortality was $28.12 \%$. There was no significant association between dialysis and survival rate.

Conclusion: Acute Kidney Injury contributes to the morbidity and mortality of hospitalized patients as well as a burden of cost to the community at large. If anticipated, it is easily prevented and if detected early, it can be skilfully treated and reversed.

\section{KEYWORDS : Acute Kidney Injury, Dialysis, Serum Creatinine}

\section{INTRODUCTION}

Acute Kidney Injury (AKI) is a state characterised by a sudden impairment of renal function resulting in accumulation of nitrogenous and other waste products normally excreted by the kidney. ${ }^{1}$ It encompasses a spectrum that ranges from minor changes in markers of renal function to the requirement for renal replacement therapy (RRT), and includes both direct injury to the kidney as well as acute impairment of function. ${ }^{2}$

Though previously known as Acute Renal Failure (ARF), AKI differs from ARF as it includes less severe conditions as well, which by virtue of being included, benefits by early intervention. The first description of AKI, then termed ischuria renalis, was by William Herberden in 1802. In 1909 it was named Acute Bright's Disease, described as a consequence of toxins, pregnancy, burns, trauma etc. and during the $1^{\text {st }}$ world war, it was named War nephritis. However, Homer W. Smith is credited for the term Acute Renal Failure in $1951^{2}$

Prevention is the key to avoid the heavy burden of mortality /morbidity associated with AKI. Most etiologies of AKI can be prevented by timely interventions at individual, community, regional and hospital levels. Increase in community wide awareness results in preventive strategies, early recognition and management.

\section{AIMS AND OBJECTIVES:}

To study the spectrum of AKI in the general medicine wards of a tertiary care hospital.

\section{MATERIALS AND METHODS}

This study is a case series of 100 patients from the General Medicine Wards of Goa Medical College between March 2014 - July 2015, who were admitted primarily for AKI or developed it while admitted for other illnesses. Eligible cases were included retrospectively after consent and followed up prospectively until the point of discharge/death. Each patient was studied in detail by taking a history, conducting an examination and laboratory assessment with complete hemogram, Renal function tests, Urine routine and microscopy, Uric acid, and additional relevant investigations like serum amylase, CPKMB, etc which were done as per the individual case requirements.

\section{INCLUSION CRITERIA}

Rise in serum creatinine (SC) of $0.3 \mathrm{mg} / \mathrm{dl}$ in $48 \mathrm{hrs}$ or $1.5 \mathrm{md} / \mathrm{dl}$ in a week from baseline

\section{EXCLUSION CRITERIA}

- Chronic kidney disease

- Acute on CKD

- Less than 12 yrs. of age

- Post Renal/Obstructive causes of AKI

\section{STATTISTICAL ANALLYSIS}

Statistical analysis was done using Microsoft excel and hand calculator. Results were expressed in the form of means, ranges and percentages.

\section{RESULTS:}

1) Age distribution: The mean age was 44.24 years and median age 46.5 years. A bimodal distribution was noted with maximum cases seen in the age groups $25-34$ and $45-64$ years.

Table 1: Age distribution among the study subjects *

\begin{tabular}{|c|c|}
\hline Age & Number of AKI patients \\
\hline $15-24$ & 9 \\
\hline $25-34$ & 21 \\
\hline $35-44$ & 16 \\
\hline $45-54$ & 20 \\
\hline $55-64$ & 20 \\
\hline $65-74$ & 8 \\
\hline $75-84$ & 6 \\
\hline Total & 100 \\
\hline
\end{tabular}

2) Sex Distribution: Out of 100 AKI patients, 78 were male and 22 were female. The male to female ratio was 3.5:1.

3)_Cause of AKI: 36 cases were due to a renal cause, 30 due to a pre-renal cause and 34 due to a combined pre-renal and renal cause. Drugs (29\%) and Acute Gastroenteritis (25\%) were among the most common pre-renal causes. Sepsis (71\%) was the most common renal cause followed by Druginduced AKI (13\%) and snake bites (7\%). Pneumonia, Malaria and skin infections were the most common infections associated with AKI. 
Table 2: Distribution of Pre-renal, Renal and Infective causes of AKI *

\begin{tabular}{|c|c|c|c|c|}
\hline Pre-Renal Etiologies & Number & Renal Etiologies & Number & Infective Etiologies \\
\hline Acute Gastro Enteritis & 15 & AGN & 2 & Pneumonia \\
\hline Haemorrhage & 2 & Trauma & 1 & Viral Syndrome \\
\hline Cardio-renal & 8 & Vasculitis & 2 & SBP \\
\hline Hepatorenal & 12 & Malignancy & 2 & UTI \\
\hline Acute pancreatitis & 6 & Snake Bite & 5 & AGE \\
\hline Drugs & 18 & Sepsis/Infections & 50 & Skin infections, cellulitis \\
\hline & & Drugs & 9 & CNS infection \\
\hline & & & & Malaria \\
\hline
\end{tabular}

\section{4) Mortality}

$27(28.12 \%)$ of the 96 patients did not survive. 6 patients were discharged against medical advice. Among them, 2 were included as survivors as their serum creatinine had normalised prior to the discharge. The remaining 4 were not included in the calculation of mortality. The distribution of cause of AKI in survivors and non-survivors is given in Table 3.

Table 3: Mortality distribution of causes of AKI among the study subjects

\begin{tabular}{|c|c|c|}
\hline Cause of AKI & Survivors & Non-survivors \\
\hline Both & 18 & 15 \\
\hline Pre - Renal & 25 & 5 \\
\hline Renal & 26 & 7 \\
\hline Total & 69 & 27 \\
\hline
\end{tabular}

\section{5) Dialysis}

Out of 28 patients who needed dialysis, 26 were dialysed, 1 refused and 1 expired prior to the procedure. Of the 26,8 were dialysed for a pre-renal cause, 10 for a renal cause and 8 for $a$ combined, prerenal and renal cause. To determine the association of dialysis and survival, data from the 96 patients who were analysed in the mortality calculation were considered. Notably, of the remaining 4 who got discharged against medical advice and were subsequently excluded from the calculation, 3 were dialysed and 1 refused dialysis.

Chi-square test was used to ascertain the association between dialysis and survival and was found to be $\chi^{2}=0.6632$, $\mathrm{p}=0.415$, which was not statistically significant.

Table 4: Dialysis among survivors and non-survivor study subjects

\begin{tabular}{|c|c|c|c|}
\hline Outcome & Dialysed & Not dialysed & Total \\
\hline Survivors & 15 & 54 & 69 \\
\hline Non-survivors & 8 & 19 & 27 \\
\hline Total & 23 & 73 & 96 \\
\hline
\end{tabular}

\section{DISCUSSION OF RESULTS}

Age:

In our study, the maximum number of AKI cases were in the age groups of 25-34 and 55-64, which correlated with a similar prospective study of 120 patients with AKI conducted in SDM

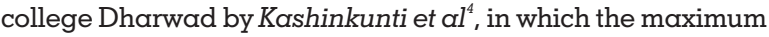
cases of AKI cases were in the age group of 31-50 years.

The median age of 46.5 years observed in our study correlated with a retrospective study on 271 AKI patients by Fon Yang et $\alpha l^{5}$ which revealed a median age of 54 years.

Another prospective study of 212 AKI patients by Wijewickrama et $a l^{6}$ in a medical ICU over 6 months in Sri Lanka (National Hospital Colombo) had a mean age of 47.8 years which is similar to our study in which the mean age was 44.24 years.

Contrary to our study findings, a retrospective study conducted in a South Indian tertiary care hospital by Eswarappa et $\alpha l^{7}$, on 500 adults critically ill patients with AKI showed maximum cases in the 61-70 years age group. A UK based retrospective study by Tariq Ali et al ${ }^{8}$ of $474 \mathrm{AKI}$ patients documented a median age of 76 years. This difference could be attributed to the larger sample size and difference in demographic characteristics in these two studies.

Sex distribution:

The male predominance (78\%) among AKI patients in our study correlated with results from the studies by Eswarappa et $\alpha l^{7}$, Wijewickrama et $t^{6}$, Kashinkunti et $\alpha \alpha^{4} l^{4}$, Fon Yong et $\alpha l^{5}$, all of which revealed a higher percentage of male patients with AKI of $63.6 \%, 61.5 \%, 56.6 \%$ and $63.5 \%$. respectively

\section{Cause of AKI:}

Like in our study, where sepsis was found to be the most common cause of AKI, accounting for $37.8 \%$ of the cases, studies by Eswarappa et $\alpha l^{7}$, Kashinkunti et al ${ }^{4}$, Wijewickrama et $a l^{6}$ revealed sepsis was the most common cause of AKI accounting for $38.6 \%, 51.3 \%$ and $45.4 \%$ of the AKI cases respectively.

\section{Dialysis:}

Dialysis requirement was seen in $28 \%$ of the patients in our study similar to studies by Fan Yang et $a l^{5}$ and Eswarappa et al ${ }^{7}$ in which the dialysis requirements were $22.5 \%$ and $37.6 \%$ respectively. In contrast, the study by Kashinkunti et $\mathrm{al}^{4}$ reported a dialysis requirement of $51.6 \%$. This difference in requirement may be due to surgical patients with AKI being included in the study unlike in our study. Tariq et $\alpha l^{8}$ reported only $7.8 \%$ of the patients required dialysis, possibly due to different treatment protocols in this UK study.

\section{Mortality:}

Mortality was $28.12 \%$ in our study correlating well with the studies by Kashinkunti et $a l^{4}$, Tariq et $a l^{8}$ and Eswarappa et $a l^{7}$ who reported mortality rates of $28.3 \%, 32.7 \%$ and $37.6 \%$ respectively.

On the contrary, Fan Yang et $\alpha l^{5}$ reported a mortality of only $19.6 \%{ }^{5}$ and Wijewickrama et $a l^{6}$ documented a mortality of $23.41 \%$. This difference could be attributed to different treatment protocols used, which might have affected mortality.

Using chi-square test, the p-value was 0.412 revealing that there was no significant association between survival and dialysis. This indicates that dialysis decisions were taken adequately and the non-survivors probably expired due to multi-organ failure or causes other than not being dialysed.

However, the convenient random sampling, use of creatinine instead of urine output (due to unreliability of urine output charting) as a means of determining AKI and a small sample size limits the validity and robustness of our study. Long term follow up of these patients who had AKI, for the development of chronic kidney disease, is a future study prospect.

\section{CONCLUSION}

AKI, though highly prevalent and harmful, is preventable and treatable. Hence early recognition and intervention is warranted. The majority of patients in our study were managed conservatively by treating the cause, without the 
multi-organ failure or causes other than not being dialysed.

However, the convenient random sampling, use of creatinine instead of urine output (due to unreliability of urine output charting) as a means of determining AKI and a small sample size limits the validity and robustness of our study. Long term follow up of these patients who had AKI, for the development of chronic kidney disease, is a future study prospect.

\section{CONCLUSION}

AKI, though highly prevalent and harmful, is preventable and treatable. Hence early recognition and intervention is warranted. The majority of patients in our study were managed conservatively by treating the cause, without the need for dialysis. Notably, there was no significant association between dialysis and mortality rate, indicating that dialysis is not the cornerstone of the management of prerenal and renal causes of AKI.

\section{Acknowledgment:}

We would like to extend our appreciation to Dr. J.P. Tiwari, Professor and Head of Nephrology Unit, Goa Medical College, for his participation in the nephrological management of the patients, conceptualization and execution of this study.

\section{REFERENCES}

1. Kasper D., Fauci A., Hauser S.,et al (2015). Harrison's Principles of Internal Medicine; $19 \mathrm{e}$ (Vol.1, No.2). McGraw-Hill.

2. John Kellum, Norbert Lameire, Peter Aspelin, et al. Kidney Disease: Improving Global Outcomes (KDIGO) Acute Kidney Injury Work Group KDIGO Clinical Practice Guideline for Acute Kidney Injury. Kidney Int Suppl 2012; 2:1-138

3. Li PK, Burdmann EA, Mehta RL.Acute Kidney Injury: Global Health Alert. J Nephropathology. 2013; 2:90-97.

4. Kashinkunti MD, Dhannanjaya. Clinical Spectrum of Acute Kidney Injury- A Study from A Tertiary Care Hospital. International Journal of Pharmaceutical and Biological Research; 2013; 4: 165-169

5. Yang $\mathrm{F}$, Zhang $\mathrm{L}, \mathrm{Wu} \mathrm{H}$, et al. Clinical analysis of cause, treatment and prognosis in acute kidney injury patients. PLoS One.2014;9:e85214.

6. Eranga Wijenwickrama, Gowri Ratnayake, Chaminda Wikramaratme, et al. Incidences and Clinical Outcomes of Acute Kidney Injury In ICU: A Prospective Observational Study in Sri Lanka; BMC Res Notes 2014;7:305

7. M. Eswarappa, M. Gireesh, V. Ravi, et al. Spectrum of Acute Kidney Injury in Critically Ill Patients:A Single Center Study From South India; Indian J Nephrol 2014 Sep-Oct; 24:280-285

8. Tariq Ali, Izhar Kan, William Simpson, et al. Incidence and Outcomes in Acute Kidney Injury.J Am Soc Nephrol, 2007; 18:1292-1298 